\title{
Is chronic breathlessness less recognised and treated compared with chronic pain? A case-based randomised controlled trial
}

\author{
To the Editor:
}

Chronic breathlessness is a major cause of suffering in chronic obstructive pulmonary disease (COPD) [1]. Despite the impact on patients' daily lives, chronic breathlessness might be under-recognised and under-treated. No previous study has explored physicians' ability to identify chronic breathlessness in relation to other chronic symptoms.

Chronic pain, in contrast to chronic breathlessness [2], is a well-recognised clinical syndrome [3]. Measurement and treatment of pain is standard of care in many settings and is considered a basic human right [3]. It has been suggested that identification and optimal treatment of chronic breathlessness should also be recognised as a basic right $[4,5]$.

Evidence-based symptomatic treatment for chronic breathlessness is available, including both non-pharmacological and pharmacological interventions. The pharmacological treatment with the strongest evidence is low-dose, oral extended-release morphine [6,7]. Observational studies report that physicians remain reluctant to prescribe opioids for relief of breathlessness in COPD patients [8-11]. A recent meta-analysis reported no evidence of clinically relevant respiratory adverse effects of low-dose opioids for chronic breathlessness [12].

We aimed to test the hypotheses that compared with chronic pain, chronic breathlessness is less likely to be recognised by physicians as needing symptomatic treatment and to receive treatment with opioids in severe COPD. The secondary aim was to compare reasons for not treating patients with opioids between chronic breathlessness and pain.

This was a double-blind, randomised (1:1), controlled, parallel-group, web-based trial using hypothetical case scenarios. The study was approved by Lund University Research Ethics Committee (Dnr: 2015/596) and prospectively registered with ClinicalTrials.gov (NCT02728674). All participants gave their informed consent. Inclusion criteria were: licensed physician; treating patients with respiratory problems in clinical practice; able to understand a case description in Swedish; not on the research team and not aware of the study's design or content; and no previous participation.

The case scenario related to a patient (59 years old, former smoker, optimally treated hypertension, medication with paracetamol and nonsteroidal anti-inflammatory drug allergy) who was diagnosed with severe COPD. The patient was optimally treated with triple inhalation therapy, vaccination against Influenza and Pneumococcus spp. and individualised pulmonary rehabilitation according to current guidelines [13]. At follow-up, the patient was said to be troubled by severe ["breathlessness" or "pain"] that markedly restricted daily activities and that had remained unchanged for at least three months. The case progressively revealed more information and questions on how the physician would manage the patient. The participant had to answer each question in order to advance to the next page and would not return to or change earlier responses.

The study endpoints were assessed in four stages.

@ERSpublications

This study highlights the need for improved assessment and management of chronic breathlessness in clinical practice http://ow.ly/S7Ua30kPmjW

Cite this article as: Ahmadi Z, Sandberg J, Shannon-Honson A, et al. Is chronic breathlessness less recognised and treated compared with chronic pain? A case-based randomised controlled trial. Eur Respir J 2018; 52: 1800887 [https://doi.org/10.1183/13993003.00887-2018]. 


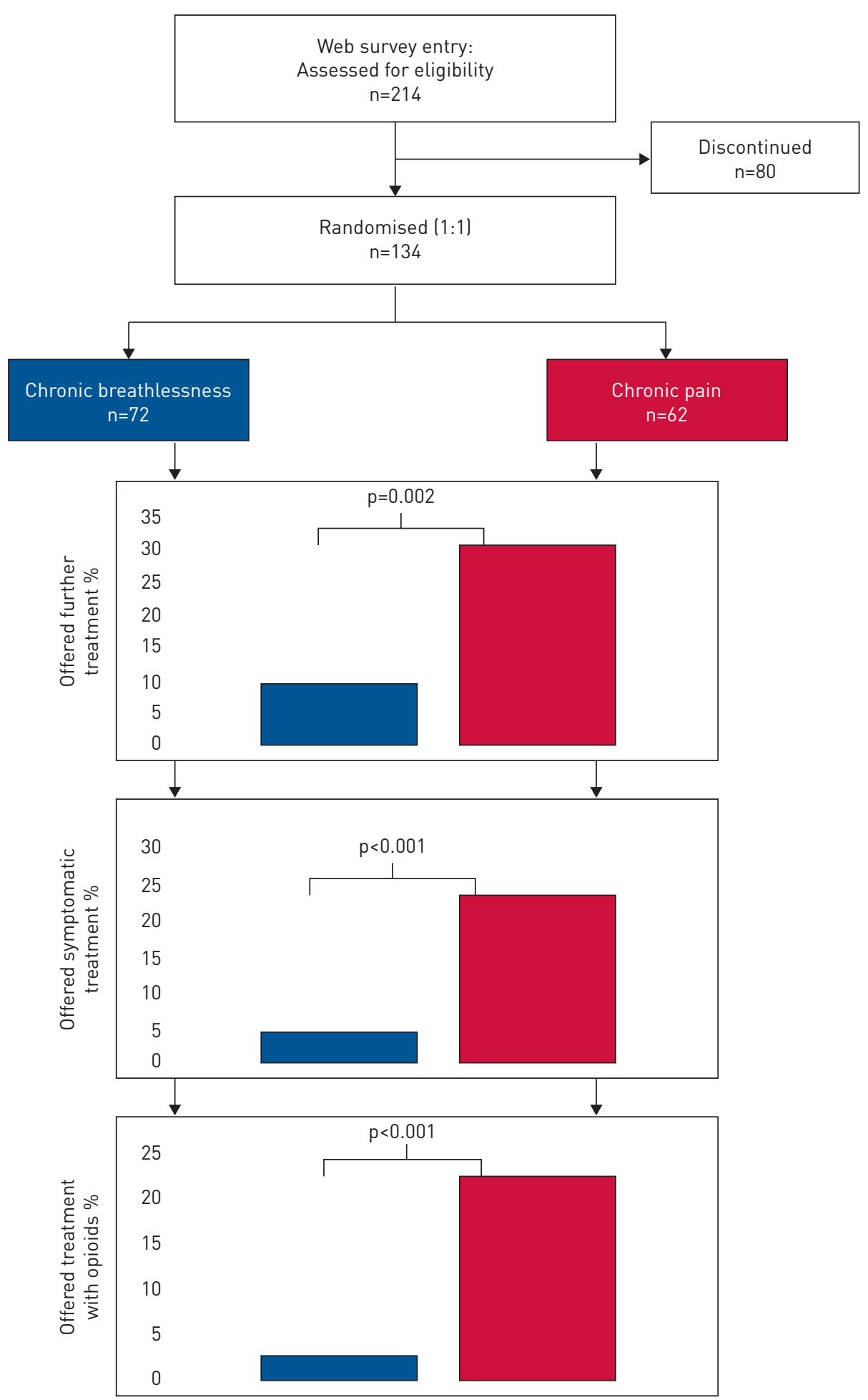

FIGURE 1 Study design and main findings.

1) Need of further treatment ("How do you manage the patient now?": "Additional diagnostic measures"; "Additional treatment"; "Active watchful waiting with follow-up visit"; or "Has optimal treatment at present, new contact if necessary”.)

2) If additional treatment, type of additional treatment ("What do you want to treat additionally in the first place?": “The COPD"; "Symptoms”; or “Other".) 
3) If symptoms, which symptomatic treatment ("Which treatments would you offer?": "Changed inhalation therapy"; "Intensified rehabilitation training"; "Benzodiazepines"; "Opioid, e.g. morphine"; "Oral steroid, e.g. prednisolone"; "Roflumilast (daxas)"; "Oxygen therapy"; "Theophylline”; or "Other”.)

4) If not opioids, reasons for not treating with opioids.

At the end of the survey, all participants were informed that the patient had breathlessness with an intensity of 7 out of 10 and answered whether the described patient suffered from chronic breathlessness, and on how often they prescribed opioids in their clinical practice for breathlessness and pain, respectively.

The endpoints were compared between groups using chi-squared tests and multivariable logistic regression adjusted for physician seniority (resident or consultant) and present working specialty (internal medicine, primary care, pulmonary medicine or other). Statistical significance was defined as a two-tailed $\mathrm{p}<0.025$ due to two co-primary endpoints. Analyses were performed using Stata version 14.2 (StataCorp LP; College Station, TX, USA). Given the observed proportions of $10 \%$ versus $30 \%$ for identifying the need for further symptomatic treatment, a sample size of 114 participants (57 per group) was required to obtain at least $80 \%$ power for the primary analysis.

From September 2016 to May 2017, a total of 134 physicians were randomised to a case with chronic breathlessness $(n=72)$ or chronic pain $(n=62$; figure 1$)$. Characteristics of the groups were well balanced; median age 42 years; $53 \%$ males; $52 \%$ worked in hospital and $46 \%$ in primary care; and the mean work experience as a physician was 11 years. For chronic breathlessness, compared with chronic pain of similar severity, significantly fewer physicians recognised the need for further treatment ( $10 \%$ versus $31 \%$; $\mathrm{p}=0.002$ ), fewer offered symptomatic treatment ( $4 \%$ versus $24 \%$; $<<0.001$ ); and markedly fewer offered treatment with opioids ( $3 \%$ versus $23 \%$; $\mathrm{p}<0.001$ ); (figure 1 ).

Findings were similar in adjusted analyses; physicians were less likely to recognise the need for further treatment (OR 0.23; 95\% CI 0.08-0.64), offer symptomatic treatment (OR $0.11 ; 95 \%$ CI $0.03-0.43$ ) and treat with opioids (OR 0.11; 95\% CI 0.02-0.51). Reasons for not treating with opioids differed markedly for breathlessness compared with pain: insufficient knowledge on usage and dosage of opioids (51\% versus $4 \% ; \mathrm{p}<0.001$ ); lack of optimal treatment guidelines ( $36 \%$ versus $17 \% ; \mathrm{p}=0.024)$; opioids considered relevant by physicians only in an end-of-life setting $(31 \%$ versus $4 \%$; $\mathrm{p}<0.001)$. However, the risk of serious adverse events was perceived as similar between the symptoms. Almost all $(\mathrm{n}=129 ; 96 \%)$ physicians considered that the patient suffered from chronic breathlessness. Fewer physicians prescribed opioids in their clinical practice for chronic breathlessness than for chronic pain $(18 \%$ versus $31 \%$; $\mathrm{p}<0.001)$ and markedly more physicians never prescribed opioids for breathlessness than pain $(47 \%$ versus $17 \%$; $\mathrm{p}<0.001)$.

This is the first randomised trial to evaluate potential under-recognition and under-treatment of chronic breathlessness in COPD. We found that compared with chronic pain, chronic breathlessness was markedly less likely to be recognised as needing symptomatic treatment and to receive treatment with opioids. The present findings extend previous small qualitative $[9,10]$ and observational data [11].

Despite that the patient was "Limited by severe ["breathlessness" or "pain"] that markedly restricted daily activities", chronic breathlessness was less likely to be identified as requiring additional treatment. There are several potential reasons for this finding. Chronic breathlessness is so common in severe respiratory disease that it might simply be considered by physicians, patients and caregivers as inevitable and part of the patient's normal life. Another potential reason is that breathlessness, despite recommendations, is rarely systematically assessed or followed-up in clinical practice [14]. The finding might also, at least partly, reflect skepticism among physicians of the availability of effective treatment for chronic breathlessness whereas pain might be considered more amenable to treatment. Main reasons for not treating with opioids were insufficient knowledge on usage and dosage of opioids and lack of optimal treatment guidelines. However, the risk of serious adverse events was perceived as similar between the symptoms.

A limitation of the study is that management decisions in clinical practice may differ from responses to a theoretical hypothetical case scenario. However, the case scenario represented a patient category and situation that is frequently encountered in the clinicians' practice, and participants were carefully instructed to answer in accordance with their usual management. The differences in symptom recognition and management in this trial were independent of the physician's working specialty and level of seniority.

For clinicians, this study highlights the need to identify symptoms and their impact more actively, and especially for chronic breathlessness; to systematically measure symptoms in daily practice and to actively consider evidence-based symptomatic treatment.

Zainab Ahmadi ${ }^{1}$, Jacob Sandberg ${ }^{2}$, Aaron Shannon-Honson ${ }^{3}$, Zac Vandersman ${ }^{3}$, David C. Currow ${ }^{3}$ and Magnus Ekström ${ }^{1,3}$ 
${ }^{1}$ Dept of Clinical Sciences, Division of Respiratory Medicine and Allergology, Lund University, Lund, Sweden. ${ }^{2}$ Center for Primary Health Care Research, Dept of Clinical Sciences in Malmö, Lund University, Lund, Sweden. ${ }^{3}$ IMPACCT, Faculty of Health, University of Technology Sydney, Ultimo, Australia.

Correspondence: Zainab Ahmadi, Dept of Clinical Sciences, Division of Respiratory Medicine and Allergology, Lund University, SE-221 00 Lund, Sweden. E-mail: zai.ahmd@gmail.com

This study is registered at ClinicalTrials.gov with identifier number NCT02728674. Additional unpublished data can be assessed by sending an e-mail to the corresponding author. To gain access, data requestors will need to sign a data access agreement.

Received: Jan 062018 | Accepted after revision: June 302018

Acknowledgements: The authors thank the Swedish Respiratory Society, the Swedish Society of Internal Medicine, and the Swedish Society of Primary Care for help with standardising recruitment, and all physicians who participated and made this trial possible.

Author contributions: Z. Ahmadi had full access to all the data in the study and takes full responsibility for the integrity of the data and the accuracy of the data analysis. Conception: M. Ekström, Z. Ahmadi; design: A. Shannon-Honson, M. Ekström, Z. Ahmadi, Z. Vandersman; acquisition of data: J. Sandberg, M. Ekström, Z. Ahmadi; analysis of data: M. Ekström, Z. Ahmadi; interpretation of data: D.C. Currow, J. Sandberg, M. Ekström, Z. Ahmadi; drafting the article: D.C. Currow, J. Sandberg, M. Ekström, Z. Ahmadi; revision for important intellectual content and approval of the version to be published: all authors. The authors affirm that the manuscript is an honest, accurate, and transparent account of the study being reported; no important aspects of the study have been omitted; and that any discrepancies from the study as planned have been explained.

Support statement: Z. Ahmadi had full access to all the data in the study and had final responsibility for the decision to submit for publication. M. Ekström was supported by unrestricted grants from The Swedish Society of Medicine, the Swedish Respiratory Society, the Swedish Heart-Lung Foundation, the Scientific Committee of Blekinge County Council, and the Swedish Society for Medical Research. The funders had no influence on the design, conduct, analysis or reporting of the study. Funding information for this article has been deposited with the Crossref Funder Registry.

Conflict of interest: None declared.

\section{References}

1 Sundh J, Ekstrom M. Persistent disabling breathlessness in chronic obstructive pulmonary disease. Int J Chron Obstruct Pulmon Dis 2016; 11: 2805-2812.

2 Johnson MJ, Yorke J, Hansen-Flaschen J, et al. Towards an expert consensus to delineate a clinical syndrome of chronic breathlessness. Eur Respir J 2017; 49: 1602277.

3 Lohman D, Schleifer R, Amon JJ. Access to pain treatment as a human right. BMC Med 2010; 8: 8.

4 Currow DC, Abernethy AP, Ko DN. The active identification and management of chronic refractory breathlessness is a human right. Thorax 2014; 69: 393-394.

5 Basoglu M. Effective management of breathlessness: a review of potential human rights issues. Eur Respir J 2017; 49: 1602099.

6 Ekstrom MP, Abernethy AP, Currow DC. The management of chronic breathlessness in patients with advanced and terminal illness. BMJ 2015; 349: g7617.

7 Ekstrom M, Nilsson F, Abernethy AA, et al. Effects of opioids on breathlessness and exercise capacity in chronic obstructive pulmonary disease. A systematic review. Ann Am Thorac Soc 2015; 12: 1079-1092.

8 Ahmadi Z, Bernelid E, Currow DC, et al. Prescription of opioids for breathlessness in end-stage COPD: a national population-based study. Int J Chron Obstruct Pulmon Dis 2016; 11: 2651-2657.

9 Young J, Donahue M, Farquhar M, et al. Using opioids to treat dyspnea in advanced COPD: attitudes and experiences of family physicians and respiratory therapists. Can Fam Physician 2012; 58: E401-E407.

10 Rocker G, Young J, Donahue M, et al. Perspectives of patients, family caregivers and physicians about the use of opioids for refractory dyspnea in advanced chronic obstructive pulmonary disease. CMAJ 2012; 184: E497-E504.

11 Janssen DJA, de Hosson SM, bij de Vaate E, et al. Attitudes toward opioids for refractory dyspnea in COPD among Dutch chest physicians. Chron Respir Dis 2015; 12: 85-92.

12 Verberkt CA, van den Beuken-van Everdingen MHJ, Schols JMGA, et al. Respiratory adverse effects of opioids for breathlessness: a systematic review and meta-analysis. Eur Respir J 2017; 50: 1701153.

13 Global Initiative for Chronic Obstructive Lung Disease. Global strategy for the diagnosis, management and Prevention of COPD 2017. Available from http://goldcopd.org Date last accessed: December 2, 2017.

14 Mularski RA, Campbell ML, Asch SM, et al. A review of quality of care evaluation for the palliation of dyspnea. Am J Respir Crit Care Med 2010; 181: 534-538. 